\title{
Realizability of Reynolds-stress turbulence models
}

\author{
U. Schumann \\ Kernforschungszentrum Karlsruhe, Institut für Reaktorentwicklung, 75 Karlsruhe, Federal Republic \\ of Germany \\ (Received 5 February 1976; final manuscript received 24 January 1977)
}

\begin{abstract}
It is shown that certain existing phenomenological models of turbulence in terms of differential equations for the Reynolds stresses $R_{a \beta}=\left\langle u_{\alpha}^{\prime} u_{\beta}^{\prime}\right\rangle$ do not guarantee realizable solutions. The known realizability conditions are $R_{a \beta} \geq 0$ for $\alpha=\beta$ and $R_{a \beta}^{2} \geq R_{a \alpha} R_{\beta \beta}$ for $\alpha \neq \beta$. A stronger requirement is that the matrix $R_{a \beta}$ be positive semi-definite. This implies three conditions like non-negative eigenvalues or non-negative principal invariants. Conditions are given which must be satisfied by the model itself in order to guarantee realizable solutions for any realizable initial and boundary conditions. Some means are proposed which can be used to change the existing models into realizable ones.
\end{abstract}

\section{INTRODUCTION}

Several phenomenological turbulence models have been proposed which consist of a set of partial differential equations for the mean velocities $U_{i} \equiv\left\langle u_{i}\right\rangle$ and the Reynolds stresses $R_{i j} \equiv\left\langle u_{i}^{\prime} u_{j}^{\prime}\right\rangle$ together with an appropriate predictive equation for the integral length scale $L$ or for the dissipation rate $\epsilon_{\circ}$ One of the most recent examples is that of Launder et al. ${ }^{1}$ These models contain closure assumptions which relate algebraically unknown correlations, such as the pressure-strain correlation $\Phi_{i j}$ $\equiv\left\langle p^{\prime}\left(u_{i, j}+u_{j, i}\right)\right\rangle$, to the known quantities $U_{i}, R_{i j}$, and $L$ or $\epsilon\left(u_{i, j} \equiv \partial u_{i} / \partial x_{j}\right)$ 。

The purpose of this paper is to show that such models may not have a solution for an arbitrary set of initial and boundary conditions in the sense that the known realizability conditions

$$
\begin{aligned}
& R_{\alpha \beta} \geq 0 \text { for } \alpha=\beta, \\
& R_{\alpha \beta}^{2} \leq R_{\alpha \alpha} R_{\beta B} \text { for } \alpha \neq \beta
\end{aligned}
$$

may be violated. (The summation convention is adopted for Latin, but not for Greek indices.) These conditions are the consequence of real velocities and Schwarz' inequality. A less known additional realizability condition is $^{2}$

$$
\operatorname{det}\left(R_{\alpha \beta}\right) \geq 0 。
$$

A proof of this condition is given in the appendix.

Equation (1a) requires nonnegative energies and (1b) states that the cross-correlation between the velocity components $u_{\alpha}^{\prime}$ and $u_{\beta}^{\prime}$ is bounded by the magnitude of autocorrelations. In order to see the physical meaning of Eq. (1c) we rewrite it in the form

$$
\begin{aligned}
R_{12} R_{23} R_{31} /\left(R_{11} R_{22} R_{33}\right) & \geq \frac{1}{2}\left[R_{12}^{2} /\left(R_{11} R_{22}\right)\right. \\
& \left.+R_{23}^{2} /\left(R_{22} R_{33}\right)+R_{31}^{2} /\left(R_{33} R_{11}\right)-1\right]
\end{aligned}
$$

from which we see that the three cross-correlations cannot take on arbitrary values. For instance, if two are well correlated with the same signs, the third must be positive. ${ }^{2}$

It appears that Eq. (1) comprises five independent inequalities. This is because of $R_{\alpha \beta}=R_{\beta \alpha}$ and (1b) implies (1a) for $\alpha=2,3$ if $R_{11} \geq 0$. We shall show, however, that it is sufficient to check three inequalities. Also, we discuss the realizability properties of the exact unclosed (unmodeled) equations for $R_{i j}$; from this we learn how to prove realizability and which terms should be modeled jointly in order to guarantee realizability. Some means will be proposed which avoid the problem of nonrealizable solutions.

The problem of nonrealizable solutions has previously been found for more complicated analytical models of turbulence. In particular, it is known that the quasinormal theory, ${ }^{3-5}$ in which the fourth-order correlations are related to second-order correlations as in a Gaussian flow, leads to the development of negative en$\mathrm{ergy}^{6-12}$ and violates other properties of turbulence. ${ }^{13-15}$ It seems, however, that this problem has not yet been fully recognized with respect to the more engineering oriented phenomenological models which close on the basis of second order correlations, Deardorff ${ }^{16}$ found, numerically, that such models sometimes produce nonrealizable solutions, but he assumed that this problem was purely a consequence of finite difference approximations. Deardorff ${ }^{16}$ and André et al. ${ }^{12}$ proposed a simple "clipping" approximation to allow feasible numerical computations with such models. (The latter actually used this approximation for triple correlations。) In this approximation one checks, after each time step and at each grid point, whether the inequalities ( $1 \mathrm{a}, \mathrm{b})$ hold and, if not, takes a new value for the correlations $R_{i j}$ corresponding to the equal sign of Eqs. (1a,b). Although this seems to be a very simple cure to the problem, it is not appealing from a theoretical point of view: If, e.g. , a negative value of $R_{11}$ is replaced by zero, the magnitude in the change of $R_{11}$ depends upon the orientation of the coordinate system. We ther efore have a nonsteady and noninvariant model.

One might object that situations where $R_{i j}$ is close to the extreme state given by the equal signs of Eq. (1) are rare in physics, and models which do not guarantee realizability might still be valid in most applications. However, then the problem remains of defining the range of applicability of a given model. Also, we should notice that the solution of the model equations is usually found by iteration and we have to insure, ther efore, that neither the initial guess nor any intermediate solution falls outside this range of applicability. In fact, it is the author's experience that such an iterative solution 
process might diverge and violate Eq. (1) although a stationary realizable solution exists. In such cases it is important to know whether the divergence is a consequence of a perhaps unstable numerical scheme, which can be adjusted, or a consequence of the model itself and of the initial and boundary conditions.

The following discussion is restricted to incompressible fluids with constant density and constant viscosity $\nu$. The averaging operator \langle\rangle denotes either the time-, space-, or ensemble mean value. Fluctuating quantities are denoted by a prime, e.g., $u_{i}^{\prime} \equiv u_{i}-\left\langle u_{i}\right\rangle$ is the fluctuating velocity component in the direction of the $i$ th coordinate.

\section{CONSEQUENCES OF THE REALIZABILITY CONDITION}

If, for some but not necessarily all values of $\alpha R_{\alpha \alpha}$ $\equiv\left\langle u_{\alpha}^{\prime} u_{\alpha}^{\prime}\right\rangle=0$, then $u_{\alpha}^{\prime}$ itself must be zero for all points in space and time over which the average \langle\rangle is taken. From the definition of $R_{\alpha \beta}$ it follows that

$$
R_{\alpha \alpha}=0 \rightarrow R_{\alpha \beta}=0, \quad \beta=1,2,3 .
$$

In cases where $\left(R_{\alpha \beta}\right)^{2}=R_{\alpha \alpha} R_{\beta B}$ for some values of $\alpha$ and $\beta(\alpha \neq \beta)$ it follows ${ }^{17}$ that $u_{\beta}^{\prime}=A u_{\alpha}^{\prime}$ where $A$ is equal at all points over which the average is taken. Consequently, we have

$$
\begin{aligned}
\left(R_{\alpha \beta}\right)^{2}=R_{\alpha \alpha} R_{B \beta} \rightarrow & R_{\alpha \beta}=A R_{\alpha \alpha} \\
R_{B \beta} & =A^{2} R_{\alpha \alpha} \\
R_{B \gamma} & =A R_{\alpha \gamma}, \quad \gamma=1,2,3 .
\end{aligned}
$$

Finally, it has been shown by du Vachat ${ }^{18} \operatorname{det}\left(R_{\alpha \beta}\right)=0$ implies $u_{\alpha}^{\prime}=A u_{\beta}^{\prime}+B u_{\gamma}^{\prime}$, where $A$ and $B$ are real constants so that

$$
\begin{aligned}
\operatorname{det}\left(R_{\alpha \beta}\right)=0-R_{\alpha \alpha} & =A R_{\alpha \beta}+B R_{\alpha \gamma}, \\
R_{\alpha \beta} & =A R_{\beta \beta}+B R_{B \gamma}, \\
R_{\alpha \gamma} & =A R_{B \gamma}+B R_{\gamma \gamma}
\end{aligned}
$$

These results will be used subsequently.

Next, we show that $\mathbf{E q}$ 。(1) is equivalent to the demand that $R_{\alpha \beta}$ is a positive semi-definite matrix so that

$$
Q \equiv x_{i} R_{i} x_{j} \geq 0
$$

for arbitrary real nonvanishing vectors $x_{i}$ (also stated by $d u$ Vachat $\left.^{18}\right)$. For proof, we note that the necessary and sufficient conditions for $Q \geq 0$ are $\mathrm{e}^{17}$

$$
\begin{aligned}
R_{11} \geq 0, & \\
R_{11} R_{22}- & R_{12}^{2} \geq 0, \\
\operatorname{det}\left(R_{\alpha \beta}\right)= & R_{11}\left(R_{22} R_{3 s}-R_{23}^{2}\right)-R_{12}\left(R_{12} R_{33}-R_{23} R_{13}\right) \\
& +R_{13}\left(R_{12} R_{23}-R_{22} R_{13}\right) \geq 0 .
\end{aligned}
$$

Therefore, if $Q \geq 0$, then Eq. (1c) and two of the conditions $(1 \mathrm{a}, \mathrm{b})$ are satisfied. Thus, Eq. (3) is necessary for Eq. (1) to be true. It is also sufficient since the remaining conditions of Eqs. $(1 \mathrm{a}, \mathrm{b})$ are consequences of Eq. (3) as can be seen: The property of positive semidefiniteness does not change under a similarity transformation $C_{0}{ }^{17}$ Therefore, we may rotate our coordinate system around the second coordinate by $90^{\circ}$ and the third coordinate by $-90^{\circ}$ so that

$$
C^{-1}\left(R_{\alpha \beta}\right) C=R_{\alpha \beta}^{\prime}=\left(\begin{array}{lll}
R_{22} & R_{23} & R_{12} \\
R_{23} & R_{33} & R_{13} \\
R_{12} & R_{13} & R_{11}
\end{array}\right) .
$$

As $R_{\alpha \beta}^{\prime}$ is positive semi-definite, the second pair of conditions $R_{22} \geq 0$ and $R_{22} R_{33}-R_{23}^{2} \geq 0$ follows from (4a) and ( $4 \mathrm{~b})$. The final pair of conditions ( $R_{33} \geq 0$ and $R_{11} R_{33}$ $-R_{13}^{2} \geq 0$ ) follows in the same manner from a rotation around the second coordinate by $-90^{\circ}$ and the third coordinate by $90^{\circ}$. This closes the required proof。

We see, that instead of the five checks required by Eq. (1), the three checks stated in (4) are sufficient. Condition (3) also implies nonnegative eigenvalues ${ }^{17}$ and nonnegative values of the three principal invariants ${ }^{17}$

$$
\begin{aligned}
I_{1} \equiv & R_{11}+R_{22}+R_{33} \geq 0, \\
I_{2} \equiv & R_{11} R_{22}-R_{12}^{2}+R_{11} R_{33}-R_{13}^{2} \\
& +R_{22} R_{33}-R_{23}^{2} \geq 0, \\
I_{3} \equiv & \operatorname{det}\left(R_{\alpha \beta}\right) \geq 0 .
\end{aligned}
$$

However, Eq. (4) is numerically most convenient.

We note that $I_{3}$ is the most sensitive measure with respect to realizability, since $I_{3}$ is positive under "normal" conditions where $R_{\alpha \alpha}>0$ and $\left(R_{\alpha \beta}\right)^{2}<R_{\alpha \alpha} R_{\beta B}(\alpha, \beta$ $=1,2,3, \alpha \neq \beta)$ and becomes zero if for any $\alpha$ and $\beta \neq \alpha$, $R_{\alpha \alpha}=0$ or $\left(R_{\alpha \beta}\right)^{2}=R_{\alpha \alpha} R_{\beta \beta}$ 。 This can easily be seen for $R_{11}=0$ or for $R_{23}^{2}=R_{2 \varepsilon} R_{33}$ from Eq. (4c) using (2a) and (2b), respectively。 For other values of $\alpha$ and $\beta$ this follows from Eq. (4c) with cyclic permuted indices。This result, in principle, implies that if $R_{\alpha B}$ varies continuously starting from a realizable initial state with $I_{3}>0$, then a nonrealizable state cannot be reached without passing through a point where $I_{3}=0$ 。 (If the average operator is the time mean value, then "initial state" means the initial guess of an iterative solution or boundary values if the model equations are parabolic and can, therefore, be solved by a marching procedure.) Moreover, $I_{3}$ is zero for all times at walls where all velocity components vanish. The single and convenient requirement

$$
I_{3}>0
$$

for all points inside the flow region away from walls is, therefore, in principle, a sufficient condition for realizability if $R_{\alpha \beta}$ is a continuous function of space and time with realizable initial conditions. Unfortunately, this result is true only if the $R_{\alpha \beta}$ are related to each other as though they were the elements of the Reynolds stress. If however, the values of $R_{\alpha \beta}$ are being generated by model equations there is no guarantee that this will be so, ${ }^{2}$ since we used Eqs. (2) which may be violated by the model results. Therefore, Eq. (6), although being sufficient in principle, is not sufficient in practice.

Finally in this paragraph, we ask for the consequences with respect to models of turbulence. We have already discussed conditions for $R_{\alpha \beta}$ itself while the Reynoldsstress models predict $D R_{\alpha \beta} / D t_{\circ} \quad D / D t$ is the total time 


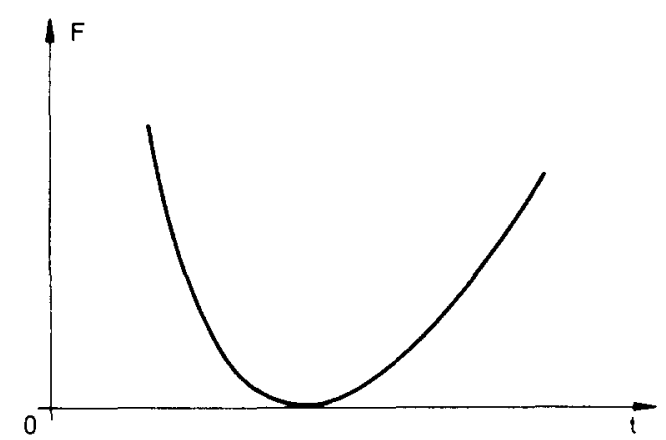

FIG. 1. Required behavior of $F$ as a function of time $t$ when approaching the limit $F=0 . \quad F$ is either $R_{\alpha \alpha}$ or $R_{\alpha \alpha} R_{\beta \beta}-R_{\alpha \beta}^{2}$, $(\alpha \neq \beta)$ or $\operatorname{det}\left(R_{\alpha \beta}\right)$.

derivative

$$
\frac{D}{D t} \equiv \frac{\partial}{\partial t}+U_{j} \frac{\partial}{\partial x_{j}} .
$$

For this purpose, we consider a point where either $R_{\alpha \alpha}$ $=0$ or $\left(R_{\alpha \beta}\right)^{2}=R_{\alpha \alpha} R_{B \beta}$ 。 If $R_{\alpha \beta}$ is a continuous function, we may use a Taylor series expansion, and as $R_{\alpha \beta}$ must be realizable in the neighborhood of the point under consideration (see $\mathrm{F}$ ig。1), it follows that for realizability $R_{\alpha \alpha}=0$ requires that

$$
\frac{\partial}{\partial t} R_{\alpha \alpha}=0, \quad \frac{\partial}{\partial x_{\gamma}} R_{\alpha \alpha}=0, \quad \gamma=1,2,3,
$$

and the first nonzero higher derivative must be positive (if $R_{\alpha \beta}$ is not a continuous function of time, then a posi-tive first time derivative is allowed)。 Similarly, if

$$
\left(R_{\alpha \beta}\right)^{2}=R_{\alpha \alpha} R_{B B},
$$

then

$$
\begin{aligned}
\frac{\partial}{\partial t}\left(R_{\alpha \beta}\right)^{2}=\frac{\partial}{\partial t}\left(R_{\alpha \alpha} R_{B \beta}\right), \quad \frac{\partial}{\partial x_{\gamma}}\left(R_{\alpha \beta}^{2}\right) & =\frac{\partial}{\partial x_{\gamma}}\left(R_{\alpha \alpha} R_{\beta \beta}\right), \\
\gamma & =1,2,3,
\end{aligned}
$$

and if

$$
\operatorname{det}\left(R_{\alpha \beta}\right)=0 \text {, }
$$

then

$$
\frac{\partial}{\partial t} \operatorname{det}\left(R_{\alpha \beta}\right)=0, \quad \frac{\partial}{\partial x_{\gamma}} \operatorname{det}\left(R_{\alpha \beta}\right)=0, \quad \gamma=1,2,3
$$

are required for realizability. These requirements are equivalent to

$$
\begin{aligned}
& R_{\alpha \alpha}=0-D R_{\alpha \alpha} / D t=0 \\
& \left(R_{\alpha \beta}\right)^{2}=R_{\alpha \alpha} R_{\beta \beta}- \\
& \quad 2 R_{\alpha \beta} D R_{\alpha \beta} / D t=R_{\alpha \alpha} D R_{\beta \beta} / D t+R_{\beta \beta} D R_{\alpha \alpha} / D t,(8 \mathrm{~b}) \\
& \operatorname{det}\left(R_{\alpha \beta}\right)=0 \rightarrow D \operatorname{det}\left(R_{\alpha \beta}\right) / D t=0
\end{aligned}
$$

for arbitrary values of $U_{j}$. Equation (8) can be used to check whether a given model (which describes $D R_{\alpha \beta} / D t$ ) has realizable solutions.

Strictly speaking, this check should be applied not only with respect to the closure assumptions but also to the numerical approximations like finite differences.

\section{REALIZABILITY OF THE EXACT (UNCLOSED) REYNOLDS-STRESS MODELS}

From the Navier-Stokes equations we obtain, as usual, ${ }^{1}$ the following equations:

$$
\frac{D R_{\alpha \beta}}{D t}=P_{\alpha \beta}+D_{\alpha \beta}^{\prime}+D_{\alpha \beta}^{\prime \prime}+D_{\alpha \beta}^{\prime \prime \prime}+\Phi_{\alpha \beta}-\epsilon_{\alpha \beta}
$$

with the

production:

$$
P_{\alpha \beta}=-R_{\alpha k} U_{\beta, k}-R_{\beta k} U_{\alpha, k} \text {, }
$$

turbulent diffusion: $\quad D_{\alpha \beta}^{\prime}=-\left\langle u_{\alpha}^{\prime} u_{\beta}^{\prime} u_{k}^{\prime}\right\rangle_{, k}$,

pressure diffusion: $\quad D_{\alpha \beta}^{\prime \prime}=-\left\langle p u_{\alpha}^{\prime}\right\rangle_{, \beta}-\left\langle p u_{\beta}^{\prime}\right\rangle_{, \alpha}$,

viscous diffusion: $\quad D_{\alpha \beta}^{\prime \prime \prime}=\nu R_{\alpha \beta, k k}$,

redistribution:

viscous dissipation: $\epsilon_{\alpha \beta}=2 \nu\left\langle u_{\alpha, k}^{\prime} u_{\beta, k}^{\prime}\right\rangle$.

These are the exact unclosed equations and we do expect, of course, that these equations will satisfy Eq. (8). In fact, we can prove this by inserting (9) in (8) and by using (2). We find that the following terms satisfy (8) independent of all other terms: $P_{\alpha \beta}, D_{\alpha \beta}^{\prime},\left(D_{\alpha \beta}^{\prime \prime}+\Phi_{\alpha \beta}\right)$, and $\left(D_{\alpha \beta}^{\prime \prime \prime}-\epsilon_{\alpha \beta}\right)$. Let us prove this, for example, for $P_{11}$ and $P_{12}$. This means that we have to prove (8) in the form

$$
R_{11}=0 \rightarrow P_{11}=-2 R_{11} U_{1,1}-2 R_{12} U_{1,2}-2 R_{13} U_{1,3}=0 .
$$

It follows from (2a) that $P_{11}=0, q_{0} e_{0} d$. In the case of $P_{12}$ we have to prove

$$
\begin{aligned}
R_{12}^{2} & =R_{11} R_{22}--2 R_{12}\left(R_{1 k} U_{2, k}+R_{2 k} U_{1, k}\right) \\
& =-2 R_{11} R_{2 k} U_{2, k}-2 R_{22} R_{1 k} U_{1, k}
\end{aligned}
$$

Here, some terms cancel and we can rewrite the condition as

$$
\left(R_{12} R_{13}-R_{11} R_{23}\right) U_{2,3}+\left(R_{12} R_{23}-R_{13} R_{22}\right) U_{1,3}=0 .
$$

Since the values of $U_{2,3}$ and $U_{1,3}$ are arbitrary, we have to prove that the terms in parentheses are zero, which, in fact, follows from (2b), q. e.d. The proof for the other terms is similar. We note, that we cannot prove realizability for $D_{\alpha \beta}^{\prime \prime}$ and $\Phi_{\alpha \beta}$ or $D_{\alpha \beta}^{\prime \prime \prime}$ and $\epsilon_{\alpha \beta}$ independently. This suggests that independent modeling of these terms may result in nonrealizable models. The fact that realizability can be proven independently for the terms in groups as indicated shows that the realizability of the exact equations is guaranteed for any sign and magnitude of the values $U_{i, j}, p$, or $\nu u_{i, j}$ 。

\section{EXAMPLES OF NONREALIZABLE TURBULENCE MODELS}

Several researchers proposed models for the redistribution or pressure-strain correlation $\Phi_{i j}$, e.g. , Rotta $^{19}$ proposed

$$
\begin{aligned}
& \Phi_{i j}=-c_{1} \epsilon\left(R_{i j}-\delta_{i j} R_{k k} / 3\right) / E, \\
& \epsilon \equiv \epsilon_{k k} / 2, \quad E \equiv R_{k k} / 2 .
\end{aligned}
$$

Naot et al. ${ }^{20}$ and Launder ${ }^{21}$ proposed

$$
\Phi_{i j}=-c_{2}\left(P_{i j}-\delta_{i j} P_{k k} / 3\right),
$$

and Crow, ${ }^{22}$ Lilly, ${ }^{23}$ and Deardorff ${ }^{18}$ include a term of 
the form

$$
\Phi_{i j}=-c_{3} R_{k k}\left(U_{i, j}+U_{j, i}\right)
$$

while Rotta ${ }^{19}$ and Launder et al. ${ }^{1}$ use

$$
\Phi_{i j}=a_{l j}^{m i} U_{l, m}
$$

instead of (10b); here, $a_{l j}^{m i}$ is a linear function of $R_{\alpha \beta}$. Usually, certain combinations of these models are used.

The coefficients $c_{i}, i=1,2,3$ are empirical constants. It might be noted that $(10 \mathrm{c})$ can be rigorously deduced on the assumption of local isotropy and (10d) follows from the exact equations by use of a truncated Taylor-series expansion. Both are based, therefore, on the assumption that the departure of the isotropic and homogeneous state is not too large. For the following demonstration of nonrealizable models we restrict ourselves to homogeneous turbulence so that the diffusion terms may be neglected.' We notice that we also need specific models for the dissipation. A model which combines the usual proposals is

$$
\epsilon_{i f}=2 \epsilon\left[d R_{i j} / R_{k k}+(1-d) \delta_{i j} / 3\right],
$$

where $0 \leq d \leq 1$ is at least a function of the Reynolds number and probably of $\operatorname{det}\left(R_{\alpha \beta}\right)$. The total dissipation $\epsilon$ itself may be specified as a function of $E, L$, and $\nu$. Using Eqs. (2) and (8) we find the following results for these models: Equation (10a) together with Eq. (11) gives a realizable model if

$$
c_{1} \geq 1-d \text {. }
$$

(This requirement has been found by Rotta ${ }^{24}$ for $d=0$.) Strictly speaking, Eq. (8) requires that $c_{1}=(1-d)$. If $c_{1}>(1-d)$, then realizability is enforced by the model stronger than required mathematically in the sense that

$$
\begin{gathered}
R_{\alpha \alpha}=0-\partial R_{\alpha \alpha} / \partial t>0, \quad \alpha=1,2,3 \\
\left(R_{\alpha \beta}\right)^{2}=R_{\alpha \alpha} R_{\beta \beta} \rightarrow 2 R_{\alpha \beta} \frac{\partial R_{\alpha \beta}}{\partial t}<R_{\alpha \alpha} \frac{\partial R_{\beta \beta}}{\partial t}+R_{\beta B} \frac{\partial R_{\alpha \alpha}}{\partial t}, \\
\beta=1,2,3 。 \beta \neq \alpha .
\end{gathered}
$$

We may call such models "over-realizable." Such models would be unsuitable for extremely anisotropic turbulence like the quasi-two-dimensional situations expected, e.g., for certain atmospheric or magnetohydrodynamic flow problems. ${ }^{25}$

Equation (10b) does not guarantee realizability. If, for example, we assume $i=1, j=1$, then-for $R_{11}=0$-we require

$$
P_{11}-c_{2}\left(P_{11}-\frac{1}{3} P_{k k}\right)-\epsilon_{11} \geq 0
$$

which is equivalent to

$-\frac{3}{2} c_{2}\left(R_{22} U_{2,2}+R_{23} U_{2,3}+R_{32} U_{3,2}+R_{33} U_{3,3}\right)-\epsilon_{11} \geq 0$

which is violated, e.g., for large positive values of $R_{22} U_{2,2}+R_{33} U_{3,3}$. Similarly, Eq. (10c) violates the realizability condition, $e_{\circ} g_{\circ}$, for $R_{11}=0$ unless $U_{1,1}$ is sufficiently large, and Eq. (10d) also violates the realizability condition for certain values of $U_{i, j}$.

\section{METHODS OF INSURING REALIZABILITY}

One way to circumvent the problem of nonrealizability consists in "clipping" as described in the introduction.
A better method, because it is continuous and tensorially invariant, might be the following: First, we split our model in two parts. One contains all the terms for which we can prove realizability [e.g., (10a) and (11) with (12)]; the other part contains all terms which do not guarantee Eq. (8). Then, we multiply the second part by a function $F(y)$ which is dependent on a measure $y$ of how close we are to the limits allowed by Eq. (1), and $F$ is zero if we reach the limits, but otherwise positive and close to unity.

The result including $\mathrm{Eq}$. (6) and the consequence that $I_{1}>0$ as long as $I_{3} \geq 0$ and $R_{\alpha \alpha}>0$ for at least one index $\alpha$ suggests the use of

$$
y=I_{3} /\left(I_{1} / 3\right)^{3} \geq 0
$$

as the required measure. ( $y=1$ for the isotropic state. ) Possible forms of the function $F(y)$ are

$$
F(y)=y^{A} \quad \text { or } \quad F(y)=1-(1-y)^{A}
$$

where the constant $A>0$ controls the steepness in the decrease of the nonrealizable terms if $y=0$ is approached. The second proposal has the advantage that for $A>1$ $\partial F / \partial y=0$ for $y=1$ so that the effect of this correction is negligible in the neighborhood of the isotropic state. The constant $A$ must be chosen large enough in order to insure realizability, since $I_{3} \geq 0$ is necessary but not sufficient for realizability of the model result.

Instead of this artifice it would, of course, be preferable if all parts of the model were constructed in such a way that realizability is always guaranteed. This is a demand which should be satisfied by closure assumptions in addition to the requests usually stated ${ }^{26}$ of correct dimensions, tensorial form, and correct properties of invariance.

\section{ACKNOWLEDGMENTS}

The author is grateful to Dr. J. L。 Lumley and Dr。 R, du Vachat for several important comments. They pointed out to me that Eq. (1c) is necessary for realizability. Also, the comments by Dr. T. Malmberg and Dr. E. G. Schlechtendahl are highly appreciated.

\section{APPENDIX}

Here, we shall prove that $\mathrm{Eq}$. (1c) is a consequence of real velocities. For this purpose we refer to the fact that $R_{i j}$ is defined as some average, e.g.

$$
R_{i j}=\lim _{N \rightarrow \infty} R_{i j}(N) ; \quad i=1,2,3 ; j=1,2,3
$$

with

$$
\begin{aligned}
& R_{i j}(N)=\sum_{k=1}^{N} w_{k} u_{i}^{\prime}\left(\mathbf{x}_{k}\right) u_{j}^{\prime}\left(\mathbf{x}_{k}\right) / W_{N}, \\
& W_{N}=\sum_{k=1}^{N} w_{k} ; \quad\left(w_{k}>0 \quad \text { for all } k>0\right),
\end{aligned}
$$

over products of the velocities $u_{i}^{\prime}\left(x_{k}\right)(i=1,2,3)$ at $N$ different points $x_{k}$ in phase space using positive weights $w_{k}$. $\quad R_{i j}$ and $\operatorname{det}\left(R_{i j}\right)$ are real values because of $w_{k}$ and $u_{i}^{\prime}$ being real. We shall show that the average $R_{i j}(N)$ satisfies 


$$
\operatorname{det} R_{i j}(N) \geq 0
$$

for all $N \geq 1$.

The proof is by complete induction. For this we first note that $\mathrm{Eq}$. (A2) is true for $N=1$ since by algebra using (A1) we find

$$
\operatorname{det} R_{i j}(1)=0 \text {. }
$$

Let us assume that (A2) is true for some specific value, $N$. Then, we have to prove that

$$
\operatorname{det} R_{i j}(N+1) \geq 0 \text { 。 }
$$

Now, using (A1),

$$
\begin{aligned}
& \operatorname{det} R_{i j}(N+1) \\
& \quad=\operatorname{det}\left\{\left[W_{N} R_{i j}(N)+w_{N+1} u_{i}^{\prime}\left(\mathbf{x}_{N+1}\right) u_{j}^{\prime}\left(\mathbf{x}_{N+1}\right)\right] / W_{N+1}\right\} 。
\end{aligned}
$$

For brevity we use the abbreviations

$$
\begin{aligned}
& P_{i j}=W_{N} R_{i j}(N) / W_{N+1}, \\
& v_{i}=u_{i}^{\prime}\left(\mathbf{x}_{N+1}\right)\left(w_{N+1} / W_{N+1}\right)^{1 / 2},
\end{aligned}
$$

and note that because of $w_{k} \geq 0$,

$$
\operatorname{det}\left(P_{i j}\right) \geq 0
$$

if (A2) holds, and that the $v_{i}$ are real values if the velocities $u_{i}^{\prime}$ are real. We then have to show that

$$
\operatorname{det}\left(P_{i j}+v_{i} v_{j}\right) \geq 0 \text {. }
$$

By algebra we find

$$
\begin{aligned}
\operatorname{det}\left(P_{i j}+v_{i} v_{j}\right)= & P_{11} P_{22} P_{33}-P_{11} P_{23}^{2}-P_{22} P_{13}^{2}-P_{33} P_{12}^{2}+2 P_{12} P_{23} P_{13} \\
& +\left(P_{22} P_{33}-P_{23}^{2}\right) v_{1}^{2}+\left(P_{11} P_{33}-P_{13}^{2}\right) v_{2}^{2}+\left(P_{11} P_{22}-P_{12}^{2}\right) v_{3}^{2}+2\left(P_{23} P_{13}-P_{33} P_{12}\right) v_{1} v_{2} \\
& +2\left(P_{12} P_{23}-P_{22} P_{13}\right) v_{1} v_{3}+2\left(P_{12} P_{13}-P_{11} P_{23}\right) v_{2} v_{3}=\operatorname{det}\left(P_{i j}\right)+Q
\end{aligned}
$$

with the quadratic expression

$$
Q=\left(v_{1}, v_{2}, v_{3}\right) \cdot M \cdot\left(v_{1}, v_{2}, v_{3}\right)^{T} .
$$

The matrix $M$ is

$$
M=\left[\begin{array}{ccc}
\left(P_{22} P_{33}-P_{23}^{2}\right) & \left(P_{23} P_{13}-P_{33} P_{12}\right) & \left(P_{12} P_{23}-P_{22} P_{13}\right) \\
\left(P_{23} P_{13}-P_{33} P_{12}\right) & \left(P_{11} P_{33}-P_{13}^{2}\right) & \left(P_{12} P_{13}-P_{11} P_{23}\right) \\
\left(P_{12} P_{33}-P_{22} P_{13}\right) & \left(P_{12} P_{13}-P_{11} P_{23}\right) & \left(P_{11} P_{22}-P_{12}^{2}\right)
\end{array}\right] .
$$

For arbitrary real values $\left(v_{1}, v_{2}, v_{3}\right)$ we have $Q \geq 0$ since $M$ is positive semi-definite, which follows from ${ }^{17}$ :

(a) $M_{11} \geq 0$ because of $\mathrm{Eq}$. (1b);

(b) $M_{11} M_{22}-M_{12}^{2}=P_{33} \operatorname{det}\left(P_{i j}\right) \geq 0$ because of Eq. (A4), (1a), and (A2);

(c) Finally by algebra, we find

$$
\operatorname{det}\left(M_{i j}\right)=\operatorname{det}\left(P_{i j}\right)^{2} \geq 0 \begin{aligned}
& \text { because of }(\mathrm{A} 4) \text { and } R_{i j} \text { being } \\
& \text { real. }
\end{aligned}
$$

Therefore, $\operatorname{det}\left[R_{i j}(N+1)\right] \geq 0$ if $\operatorname{det}\left[R_{i j}(N)\right] \geq 0$ and the latter is true for all $N$ because it is true for $N=1$. Thus, Eq. (1c) is a condition which must be satisfied by $R_{i j}$ if this Reynolds stress tensor describes a real flow.

'B. E. Launder, G. J. Reece, and W. Rodi, J. Fluid Mech. 68,537 (1975).

${ }^{2}$ J. L. Lumley (personal communication).

${ }^{3}$ M. Millionshchikov, C. R. Acad. Sci. USSR 32, 615 (1941).

${ }^{4}$ I. Proudman and W. H. Reid, Philos. Trans. R. Soc. London A 247, 163 (1954).

${ }^{5}$ T. Tatsumi, Proc. R. Soc. London A 239, 16 (1957).

${ }^{6}$ E. E. O'Brien and G. C. Francis, J. Fluid Mech. 13, 369 (1962).

${ }^{7}$ Y. Ogura, Phys, Fluids 5, 395 (1962).
${ }^{8} \mathrm{Y}$. Ogura, J. Fluid Mech. 16, 33 (1963).

${ }^{9} \mathrm{R}$. H. Kraichnan, in Hydrodynamic In stability (American Mathematical Society, Providence, R. I. , 1962), p. 199.

${ }^{10} \mathrm{H}$. Tanaka, J. Meteorol. Soc. Jpn. 47; 373 (1969).

${ }^{11}$ S. A. Orszag, Phys. Rev. Lett. 16, 441 (1966).

${ }^{12} \mathrm{~J}$. C. André, G. DeMoor, P. Lacarrère, and R. du Vachat, J. Atmos. Sei, 33, 476 (1976).

${ }^{13}$ R. Betchov, J. Fluid Mech. 1, 497 (1956).

${ }^{14} \mathrm{~S}$. A. Orszag, J. Fluid Mech. 41, $363(1970)$.

${ }^{15} \mathrm{~S}$. A. Orszag, in Proceedings of the 1973 Les Houches Summer School on Theoretical Physics (Gordon and Breach, New York, 1977) (to be published).

${ }^{16}$ J. W. Deardorff, J. Fluids Eng. (Trans. ASME) 95, 429 (1973)

${ }^{17}$ R. Zurmühl, Matrizen (Springer-Verlag, Berlin, 1964), 4th ed. , pp. 26, 130, 158, 160 .

${ }^{18} \mathrm{R}$. du Vachat, Phys. Fluids 20, 551 (1977).

${ }^{19}$ J. Rotta, Z. Phys. 129, 547 (1951).

${ }^{20} \mathrm{D}$. Naot, A. Shavit, and M. Wolfshtein, Israel J. Technol. $8,259(1970)$.

${ }^{21}{ }^{3}$. E. Launder, J. Fluid Mech. 67, 569 (1975).

${ }^{22}$ S. C. Crow, J, Fluid Mech. 33, 1 (1968).

${ }^{23} \mathrm{D}$. K. Lilly, in Proceedings of the IBM Scientific Computing Symposium on Environmental Sciences (IBM, White Plains, N. Y., 1967), p. 195.

${ }^{24}$ J. C. Rotta, Turbulente Strömungen (Teubner, Stuttgart, 1972), p. 124.

${ }^{25}$ U. Schumann, J. Fluid Mech. 76, 31 (1976).

${ }^{26}$ C. du P. Donaldson, AIAA J. 10,4 (1972). 